\title{
Simulação de reações iônicas empregando modelo quântico
}

\author{
Vinicius G. Ribeiro \\ FACIN - UniRitter \\ vinicius@uniritter.edu.br jorge.zabadal@ufrgs.br \\ Jorge Zabadal \\ DEMEC - UFRGS
}

\author{
Michele G. \\ Salgueiro \\ CEMC - UCS \\ mgsalgueiro@ucs.br
}

\author{
Edson Chiaramonte \\ CEE - UERS \\ edsonchi@portoweb.com.br
}

\section{Resumo}

O presente trabalho apresenta um novo método analítico para resolver a equação de Schrödinger empregando computação algébrica. O método é baseado em transformações de Bäcklund, as quais fornecem soluções de forma fechada para a função onda contendo funções arbitrárias de argumentos conhecidos. Essa nova formulação provê redução significante no tempo de processamento necessário para obter resultados numéricos, permitindo efetuar simulações de reações químicas em tempo real. É apresentada a simulação de um cenário físico envolvendo a formação de uma ligação iônica química entre Flúor e Potássio, para representar a correspondente dinâmica molecular.

\begin{abstract}
This work presents a new analytical method for solving the time dependent Schrödinger equation. The method is based on Bäcklund transformations, which furnishes closed form solutions for the wave function as variety containing arbitrary functions of known arguments. This formulation provides a significant reduction on the time processing required to obtain numerical results, allowing carry out real-time simulations of chemical reactions. A physical scenario involving the formation of an ionic chemical bond between Fluor and Potassium is reported, in order to sketch the corresponding molecular dynamics.
\end{abstract}

\section{Introdução}

A simulação de reações químicas consiste na previsão de mecanismos, compostos intermediários e produtos de reação, uma vez conhecidos os reatantes, os catalisadores e as condições às quais está sujeito o meio reativo (temperatura e pressão).

A previsão de mecanismos e produtos de reação segue basicamente duas estratégias bastante distintas. A primeira, essencialmente qualitativa, consiste no emprego de regras empíricas e semi-empíricas oriundas da química clássica, enquanto a segunda, de natureza quantitativa, é baseada no uso de modelos quânticos.

Ocorre que os poucos sistemas de simulação comerciais concebidos especificamente para essa aplicação também resultam computacionalmente onerosos, embora baseados em formulações variacionais que fornecem soluções aproximadas, implementadas com o intuito de viabilizar seu emprego em microcomputadores. Isto ocorre porque a densidade eletrônica é calculada a partir da função de onda correspondente a cada elétron presente no sistema reativo, o que torna o processo de simulação inviável até mesmo para moléculas contendo pouco mais de dez átomos.

Apesar de ambas as estratégias mencionadas apresentarem inconvenientes particulares quando utilizadas de forma isolada, a formulação de um modelo quantitativo que utilize informações oriundas da química clássica e dos modelos quânticos pode resultar em um sistema de simulação que reúna as vantagens de cada um deles, isto é, o tempo de processamento reduzido e a concordância com os dados experimentais.

Embora desde a década de 1950 tenham sido desenvolvidos estudos visando estabelecer uma interface entre as duas abordagens apresentadas, especificamente em uma área denominada química quântica, os avanços obtidos nessa área tem sido direcionados especificamente para a análise de problemas em regime estacionário $[1,2,3,4]$.

Em particular, a chamada Qualitative Molecular Orbital Theory (QMOT - ou Teoria Qualitativa do Orbital Molecular), baseada em análise energética e configuracional das moléculas, fornecem algumas regras complementares quanto à possibilidade de ocorrência de reações químicas. Infelizmente, essas regras têm sido utilizadas com sucesso apenas para estabelecer condições para as quais determinadas reações não ocorrem devido a limitações de natureza energética [5].

De maneira geral, as abordagens quantitativas desenvolvidas nessa área têm apresentado essencialmente a mesma limitação: os modelos aproximados, cujo tempo de processamento resulta suficientemente reduzido para viabilizar sua execução em computadores de pequeno porte, tem sido utilizados com sucesso apenas na resolução de problemas em regime estacionário, que fornecem como resultado propriedades moleculares de compostos isolados. Essas abordagens não permitem efetuar previsões sobre o comportamento de dois ou mais compostos quando formam uma mistura reativa.

Quanto a problemas de dinâmica molecular as formulações usualmente empregadas para simular processos reativos são baseadas nos métodos Density Function Theory (DFT). Dentre estes métodos o 
algoritmo mais eficiente do ponto de vista do tempo de processamento é denominado Método Híbrido de CarrParrinello, que é uma formulação variacional na qual o lagrangiano que descreve a dinâmica do sistema considera simultaneamente o rearranjo da nuvem eletrônica e o deslocamento dos núcleos dos átomos correspondentes [6]. Essa característica peculiar do método, embora promova uma redução significativa no tempo de processamento requerido para o cálculo da evolução temporal da função densidade eletrônica em relação aos demais métodos variacionais, ainda produz resultados em uma escala de tempo incompatível com a capacidade de processamento dos computadores pessoais disponíveis no mercado. A título de exemplo a simulação de sistemas químicos contendo cerca de 1000 átomos demanda aproximadamente uma semana de processamento para simular $10^{-12}$ segundos de reação, utilizando um cluster contendo 1232 CPUs Power4 - 1.3 GHZ [6]. É importante observar que o cluster mencionado possui uma velocidade de processamento de 5.2 teraflop por segundo. Além disso, a escala de tempo típica para que ocorra o rompimento ou a formação de ligações químicas durante o processo reativo é de cerca de $10^{-9}$ segundos. Assim, o tempo total de processamento requerido para simular o processo reativo completo, isto é, até a formação dos produtos, resulta cerca de mil vezes maior para a mesma formulação. Recentemente um novo método baseado na integral de caminho de Feynman, que também utiliza o algoritmo de CarParrinello, foi utilizado com sucesso na simulação de modificações estruturais da molécula de água, processo que exigiu cerca de cinco horas de simulação em um computador pessoal.

Em resumo, as reações químicas constituem um fenômeno tipicamente transiente, para o qual existem poucos sistemas de simulação disponíveis no mercado. Esses sistemas possuem ainda custo bastante elevado, e não são capazes de fornecer resultados em tempo hábil quanto à estrutura dos compostos intermediários e produtos de reação, mesmo para sistemas reativos contendo um número bastante reduzido de átomos. É importante ressaltar que os sistemas químicos que constituem o objeto de estudo da simulação de reações químicas envolvem, via de regra, dezenas de átomos.

O trabalho proposto consiste na elaboração de um sistema de simulação baseado em uma solução analítica para um modelo não-linear que deriva da equação de Schrödinger dependente do tempo, e descreve o potencial de interação entre os átomos presentes no sistema reativo. A solução é obtida utilizando mudanças de variáveis que convertem esse modelo não-linear em uma equação diferencial linear puramente difusiva. A conversão do modelo não-linear em uma equação puramente difusiva é efetuada utilizando transformações de Bäcklund, que consiste na aplicação de funções ou operadores sobre a solução de uma determinada equação diferencial, com a finalidade de convertê-la em uma solução de outra equação diferencial de maior interesse prático.

\section{Fundamentos teóricos}

A Equação de Schrödinger é utilizada para descrever a nuvem eletrônica das moléculas, permitindo, assim, efetuar previsões de mecanismos e produtos de reação. Em outras palavras, é possível prever a formação e a ruptura de ligações, que ocorrem entre as moléculas envolvidas no processo reativo.

A equação de Schrödinger dependente do tempo é dada por

$$
-\frac{\mathrm{h}^{2}}{2 m} \nabla^{2} \Psi+V \Psi=i \mathrm{~h} \frac{\partial \Psi}{\partial t},
$$

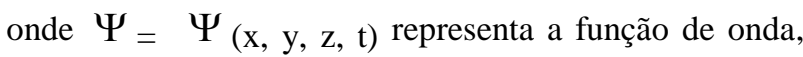
$V$, o potencial de Coulomb; $\mathrm{h}=\mathrm{h} / 2 \pi$, a constante de Planck; $m$, a massa do elétron em repouso; $i$, a unidade imaginária e $t$, o tempo. Isolando o potencial, $V$, da equação (1), obtém-se

$V=\frac{\mathrm{h}^{2}}{2 m} \frac{\nabla^{2} \Psi}{\Psi}+\frac{i \mathrm{~h}}{\Psi} \frac{\partial \Psi}{\partial t}$.

Derivando $V(\Psi)$, da equação (2), em relação as variáveis espaciais x e y, vem

$$
\frac{\partial V}{\partial x}=\frac{\partial}{\partial x}\left(\frac{\mathrm{h}^{2}}{2 m} \frac{\nabla^{2} \Psi}{\Psi}+\frac{i \mathrm{~h}}{\Psi} \frac{\partial \Psi}{\partial t}\right)
$$

e

$$
\frac{\partial V}{\partial y}=\frac{\partial}{\partial y}\left(\frac{\mathrm{h}^{2}}{2 m} \frac{\nabla^{2} \Psi}{\Psi}+\frac{i \mathrm{~h}}{\Psi} \frac{\partial \Psi}{\partial t}\right)
$$




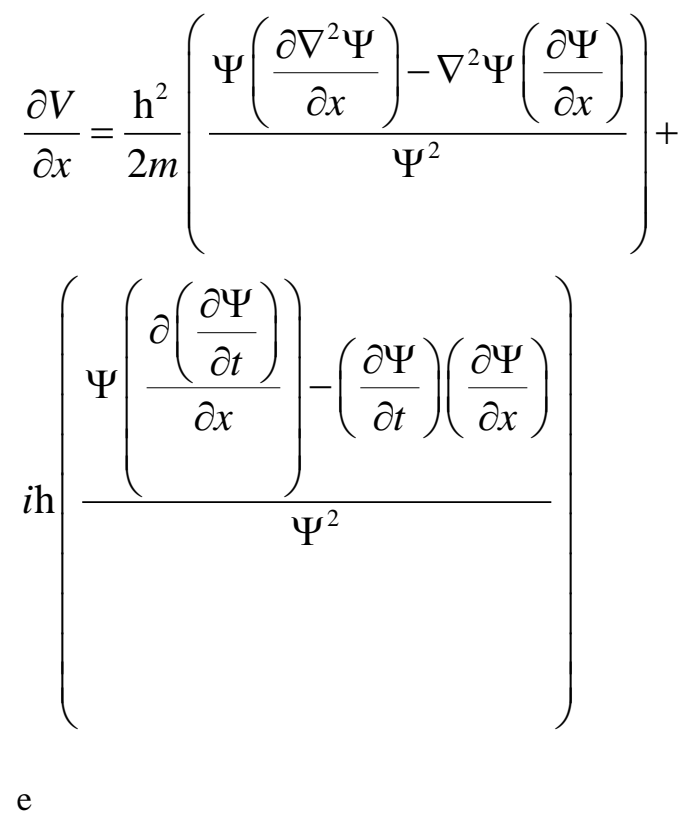

$\frac{\partial V}{\partial y}=\frac{h^{2}}{2 m}\left(\frac{\Psi\left(\frac{\partial \nabla^{2} \Psi}{\partial y}\right)-\nabla^{2} \Psi\left(\frac{\partial \Psi}{\partial y}\right)}{\Psi^{2}}\right)+$

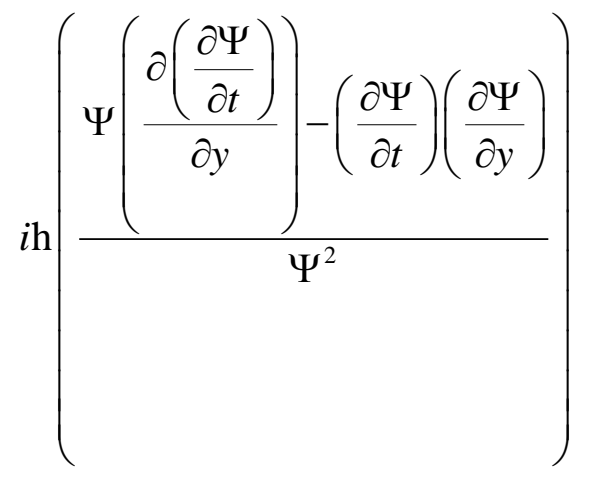

Dividindo (5) por (6), resulta
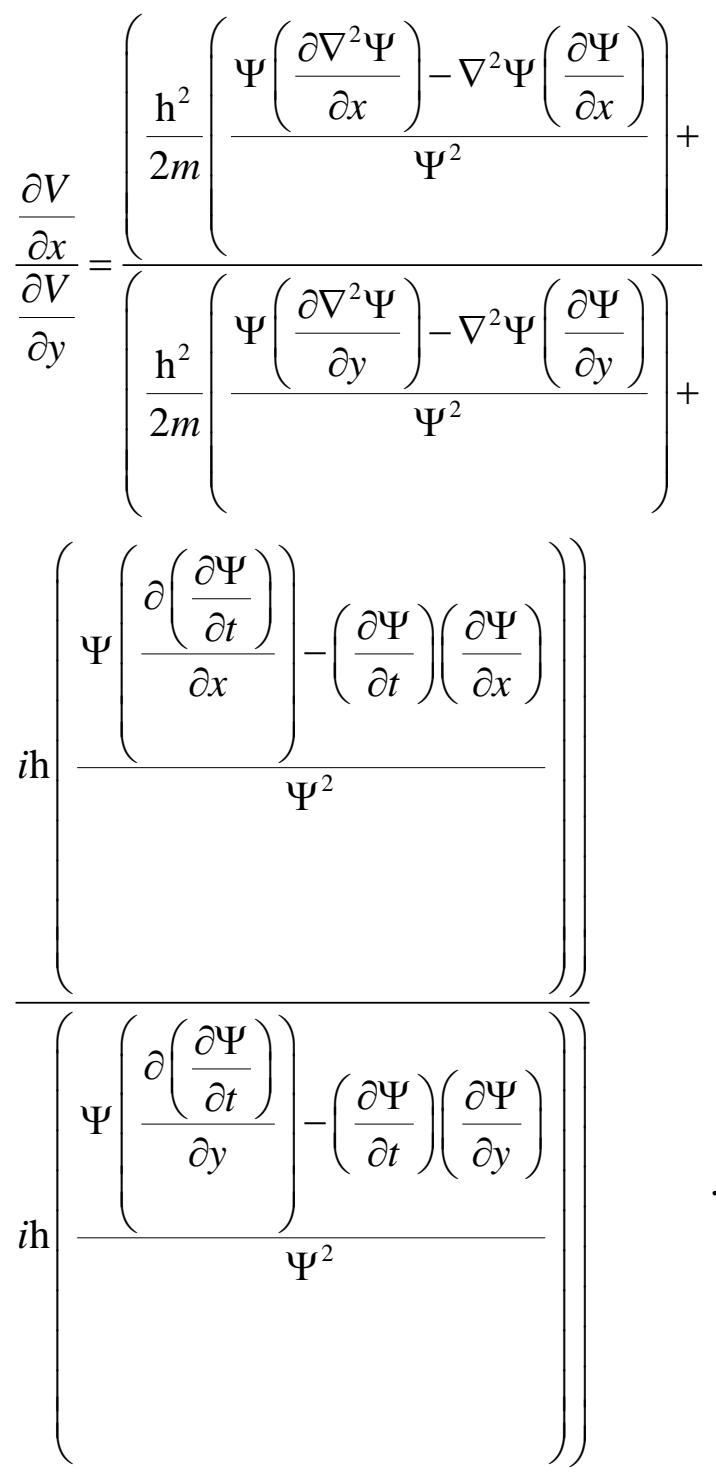

Aplicando a regra da cadeia para eliminar o potencial do membro esquerdo de (7), obtém-se

$$
\frac{\frac{\partial V}{\partial x}}{\frac{\partial V}{\partial y}}=\frac{\frac{\partial V}{\partial \Psi} \frac{\partial \Psi}{\partial x}}{\frac{\partial V}{\partial \Psi} \frac{\partial \Psi}{\partial y}}=\frac{\frac{\partial \Psi}{\partial x}}{\frac{\partial \Psi}{\partial y}} .
$$

A dependência de $V$ com $\Psi$ se deve ao fato da função representar o potencial de campo autoconsistente, que supostamente possui o mesmo conjunto de isolinhas da função de onda. Igualando as expressões (7) e (8), obtém-se:

$$
\begin{aligned}
& \frac{\partial \Psi}{\partial x}\left(\frac{\mathrm{h}^{2}}{2 m} \frac{\partial \nabla^{2} \Psi}{\partial y}+i \mathrm{~h} \frac{\partial \Psi / \partial t}{\partial y}\right)- \\
& \frac{\partial \Psi}{\partial y}\left(\frac{\mathrm{h}^{2}}{2 m} \frac{\partial \nabla^{2} \Psi}{\partial x}+i \mathrm{~h} \frac{\partial \Psi / \partial t}{\partial x}\right)=0
\end{aligned}
$$


A equação resultante é não-linear, de modo que não é possível aplicar formulações baseadas em combinações lineares de orbitais atômicos LCAO-MO.

Como uma primeira tentativa de obtenção de uma solução e forma fechada, é aplicada uma mudança de variáveis para converter a equação (9) em uma equação diferencial ordinária. Para tanto, é definida a seguinte variável auxiliar:

$u=c_{0}+c_{1} x+c_{2} y+c_{3} z+c_{4} t$

Utilizando a regra da cadeia para redefinir as derivadas presentes na equação (9), resulta:

$$
\begin{aligned}
& \frac{\partial \Psi}{\partial x}=\frac{d \Psi}{d u} \frac{d u}{d x}=c_{1} \frac{d \Psi}{d u} \\
& \frac{\partial \Psi}{\partial y}=c_{2} \frac{d \Psi}{d u} \\
& \frac{\partial \Psi}{\partial z}=c_{3} \frac{d \Psi}{d u} \\
& \frac{\partial \Psi}{\partial t}=c_{4} \frac{d \Psi}{d u}
\end{aligned}
$$

$\frac{\partial^{2} \Psi}{\partial x \partial t}=c_{1} c_{4} \frac{d^{2} \Psi}{d u^{2}}$

$\frac{\partial^{2} \Psi}{\partial y \partial t}=c_{2} c_{4} \frac{d^{2} \Psi}{d u^{2}}$

$\frac{\partial^{3} \Psi}{\partial x \partial z^{2}}=c_{1} c_{3}{ }^{2} \frac{d^{3} \Psi}{d u^{3}}$

$\frac{\partial^{3} \Psi}{\partial y \partial z^{2}}=c_{2} c_{3}{ }^{2} \frac{d^{3} \Psi}{d u^{3}}$

$$
\frac{\partial^{3} \Psi}{\partial x \partial y^{2}}=c_{1} c_{2}{ }^{2} \frac{d^{3} \Psi}{d u^{3}}
$$

$$
\frac{\partial^{3} \Psi}{\partial x^{2} \partial y}=c_{1}^{2} c_{2} \frac{d^{3} \Psi}{d u^{3}}
$$

$$
\frac{\partial^{3} \Psi}{\partial x^{3}}=c_{1}^{3} \frac{d^{3} \Psi}{d u^{3}}
$$

$\frac{\partial^{3} \Psi}{\partial y^{3}}=c_{2}{ }^{3} \frac{d^{3} \Psi}{d u^{3}}$

Substituindo as derivadas na equação (9), obtém-se

$c_{1} \frac{\partial \Psi}{\partial u}\left(\frac{\mathrm{h}^{2}}{2 m} c_{1}^{2} c_{2} \frac{d^{3} \Psi}{d u^{3}}+\frac{\mathrm{h}^{2}}{2 m} c_{2}{ }^{3} \frac{d^{3} \Psi}{d u^{3}}+\right.$

$\left.\frac{\mathrm{h}^{2}}{2 m} c_{2} c_{3}{ }^{2} \frac{d^{3} \Psi}{d u^{3}}+i \mathrm{~h} c_{2} c_{4} \frac{d^{2} \Psi}{d u^{2}}\right)-$

$c_{2} \frac{\partial \Psi}{\partial u}\left(\frac{\mathrm{h}^{2}}{2 m} c_{1}^{3} \frac{d^{3} \Psi}{d u^{3}}+\frac{\mathrm{h}^{2}}{2 m} c_{1} c_{2}{ }^{2} \frac{d^{3} \Psi}{d u^{3}}+\right.$

$\left.\frac{\mathrm{h}^{2}}{2 m} c_{1} c_{3}{ }^{2} \frac{d^{3} \Psi}{d u^{3}}+i \mathrm{~h} c_{1} c_{4} \frac{d^{2} \Psi}{d u^{2}}\right) \equiv 0$

Uma vez que a expressão resultante é identicamente nula, qualquer função do argumento u é solução exata da equação (23) e, portanto também da equação (9). Assim,

$\Psi=F_{0}\left(c_{0}+c_{1} x+c_{2} y+c_{3} z+c_{4} t\right)$,

onde $F_{0}$ representa uma função arbitrária, a ser especificada através da aplicação de uma condição inicial.

Uma vez que a função de onda, $\Psi$, é expressa como uma função arbitrária de um argumento linear nas varíaveis espacias, a validade da solução é restrita a subdomínios de pequena extensão. Isso ocorre porque as isossuperfícies de densidade eletrônica resultam quadráticas, de modo que se faz necessário discretizar o domínio em elementos cuja dimensão característica é da ordem de um comprimento típico de ligação (em torno de $1 \AA$ ).

Adotando agora uma forma quadrática para a variável $u$, e aplicando a mesma mudança de variável na equação (9), resulta

$$
\begin{aligned}
& u=c_{0}+c_{1} t+c_{2} x+c_{3} y+c_{4} z+c_{5} x y+c_{6} x z+ \\
& c_{7} y z+c_{8} x^{2}+c_{9} y^{2}+c_{10} z^{2}
\end{aligned}
$$

$$
\frac{\partial \Psi}{\partial x}=\left(c_{2}+c_{5} y+c_{6} z+2 c_{8} x\right) \frac{d \Psi}{d u}
$$

$$
\frac{\partial \Psi}{\partial y}=\left(c_{3}+c_{5} x+c_{7} z+2 c_{9} y\right) \frac{d \Psi}{d u}
$$




$$
\begin{aligned}
& \frac{\partial \Psi}{\partial z}=\left(c_{4}+c_{6} x+c_{7} y+2 c_{10} z\right) \frac{d \Psi}{d u} \\
& \frac{\partial^{3} \Psi}{\partial x \partial y^{2}}=2 c_{5}\left(c_{3}+c_{5} x+c_{7} z+2 c_{9} y\right) \frac{d^{2} \Psi}{d u^{2}} \\
& \frac{\partial \Psi}{\partial t}=c_{1} \frac{d \Psi}{d u} \\
& \frac{\partial V}{\partial x}=\frac{\mathrm{h}^{2}}{2 m}\left(\frac{\Psi\left(\frac{\partial \nabla^{2} \Psi}{\partial x}\right)-\nabla^{2} \Psi\left(\frac{\partial \Psi}{\partial x}\right)}{\Psi^{2}}\right)+ \\
& +2 c_{9}\left(c_{2}+c_{5} y+c_{6} z+2 c_{8} x\right) \frac{d^{2} \Psi}{d u^{2}}+\left(c_{2}+c_{5} y+\right. \\
& \left.c_{6} z+2 c_{8} x\right)\left(c_{3}+c_{5} x+c_{7} z+2 c_{9} y\right)^{2} \frac{d^{3} \Psi}{d u^{3}} \\
& \frac{\partial^{3} \Psi}{\partial x^{2} \partial y}=2 c_{5}\left(c_{2}+c_{5} y+c_{6} z+2 c_{8} x\right) \frac{d^{2} \Psi}{d u^{2}}+ \\
& 2 c_{8}\left(c_{3}+c_{5} x+c_{7} z+2 c_{9} y\right) \frac{d^{2} \Psi}{d u^{2}}+\left(c_{2}+c_{5} y+\right. \\
& \left.c_{6} z+2 c_{8} x\right)^{2}\left(c_{3}+c_{5} x+c_{7} z+2 c_{9} y\right) \frac{d^{3} \Psi}{d u^{3}} \\
& \frac{\partial^{3} \Psi}{\partial x^{3}}=6 c_{8}\left(c_{2}+c_{5} y+c_{6} z+2 c_{8} x\right) \frac{d^{2} \Psi}{d u^{2}} \\
& +\left(c_{2}+c_{5} y+c_{6} z+2 c_{8} x\right)^{3} \frac{d^{3} \Psi}{d u^{3}} \\
& \frac{\partial^{2} \Psi}{\partial x \partial t}=c_{1}\left(c_{2}+c_{5} y+c_{6} z+2 c_{8} x\right) \frac{d^{2} \Psi}{d u^{2}} \\
& \frac{\partial^{2} \Psi}{\partial y \partial t}=c_{1}\left(c_{3}+c_{5} x+c_{7} z+2 c_{9} y\right) \frac{d^{2} \Psi}{d u^{2}} \\
& \frac{\partial^{3} \Psi}{\partial x \partial z^{2}}=2 c_{6}\left(c_{4}+c_{6} x+c_{7} y+2 c_{10} z\right) \frac{d^{2} \Psi}{d u^{2}}+ \\
& 2 c_{10}\left(c_{2}+c_{5} y+c_{6} z+2 c_{8} x\right) \frac{d^{2} \Psi}{d u^{2}}+\left(c_{2}+c_{5} y+\right. \\
& \left.c_{6} z+2 c_{8} x\right)\left(c_{4}+c_{6} x+c_{7} y+2 c_{10} z\right)^{2} \frac{d^{3} \Psi}{d u^{3}} \\
& \frac{\partial^{3} \Psi}{\partial y \partial z^{2}}=2 c_{7}\left(c_{4}+c_{6} x+c_{7} y+2 c_{10} z\right) \frac{d^{2} \Psi}{d u^{2}}+ \\
& 2 c_{10}\left(c_{3}+c_{5} x+c_{7} z+2 c_{9} y\right) \frac{d^{2} \Psi}{d u^{2}}+\left(c_{3}+c_{5} x+\right. \\
& \left.c_{7} z+2 c_{9} y\right)\left(c_{4}+c_{6} x+c_{7} y+2 c_{10} z\right)^{2} \frac{d^{3} \Psi}{d u^{3}} \\
& \begin{array}{l}
\frac{\partial^{3} \Psi}{\partial y^{3}}=6 c_{9}\left(c_{3}+c_{5} x+c_{7} z+2 c_{9} y\right) \frac{d^{2} \Psi}{d u^{2}} \\
+\left(c_{3}+c_{5} x+c_{7} z+2 c_{9} y\right)^{3} \frac{d^{3} \Psi}{d u^{3}}
\end{array} . \\
& \left(c_{2}+c_{5} y+c_{6} z+2 c_{8} x\right) \frac{\partial \Psi}{\partial u}\left[\frac { \mathrm { h } ^ { 2 } } { 2 m } \left(2 c _ { 5 } \left(c_{2}+\right.\right.\right. \\
& \left.c_{5} y+c_{6} z+2 c_{8} x\right) \frac{d^{2} \Psi}{d u^{2}}+2 c_{8}\left(c_{3}+c_{5} x+c_{7} z+\right. \\
& \left.2 c_{9} y\right) \frac{d^{2} \Psi}{d u^{2}}+\left(c_{2}+c_{5} y+c_{6} z+2 c_{8} x\right)^{2}\left(c_{3}+\right. \\
& \left.c_{5} x+c_{7} z+2 c_{9} y\right) \frac{d^{3} \Psi}{d u^{3}}+
\end{aligned}
$$




$$
\begin{aligned}
& 6 c_{9}\left(c_{3}+c_{5} x+c_{7} z+2 c_{9} y\right) \frac{d^{2} \Psi}{d u^{2}}+ \\
& \left(c_{3}+c_{5} x+c_{7} z+2 c_{9} y\right)^{3} \frac{d^{3} \Psi}{d u^{3}}+ \\
& 2 c_{7}\left(c_{4}+c_{6} x+c_{7} y+2 c_{10} z\right) \frac{d^{2} \Psi}{d u^{2}}+ \\
& 2 c_{10}\left(c_{3}+c_{5} x+c_{7} z+2 c_{9} y\right) \frac{d^{2} \Psi}{d u^{2}}+ \\
& \left(c_{3}+c_{5} x+c_{7} z+2 c_{9} y\right)\left(c_{4}+c_{6} x+c_{7} y+\right. \\
& \left.\left.2 c_{10} z\right)^{2} \frac{d^{3} \Psi}{d u^{3}}\right)+i \mathrm{~h} c_{1}\left(c_{3}+c_{5} x+c_{7} z+\right. \\
& \left.\left.2 c_{9} y\right) \frac{d^{2} \Psi}{d u^{2}}\right]-\left(c_{3}+c_{5} x+c_{7} z+2 c_{9} y\right) \frac{\partial \Psi}{\partial u} \\
& {\left[\frac { \mathrm { h } ^ { 2 } } { 2 m } \left(6 c_{8}\left(c_{2}+c_{5} y+c_{6} z+2 c_{8} x\right) \frac{d^{2} \Psi}{d u^{2}}+\right.\right.} \\
& \left(c_{2}+c_{5} y+c_{6} z+2 c_{8} x\right)^{3} \frac{d^{3} \Psi}{d u^{3}}+2 c_{5}\left(c_{3}+\right. \\
& \left.c_{5} x+c_{7} z+2 c_{9} y\right) \frac{d^{2} \Psi}{d u^{2}}+2 c_{9}\left(c_{2}+c_{5} y+c_{6} z+\right. \\
& \left.2 c_{8} x\right) \frac{d^{2} \Psi}{d u^{2}}+\left(c_{2}+c_{5} y+c_{6} z+2 c_{8} x\right) \\
& \left(c_{3}+c_{5} x+c_{7} z+2 c_{9} y\right)^{2} \frac{d^{3} \Psi}{d u^{3}}+2 c_{6}\left(c_{4}+c_{6} x+\right. \\
& \left.c_{7} y+2 c_{10} z\right) \frac{d^{2} \Psi}{d u^{2}}+2 c_{10}\left(c_{2}+c_{5} y+c_{6} z+\right. \\
& \left.2 c_{8} x\right) \frac{d^{2} \Psi}{d u^{2}}+\left(c_{2}+c_{5} y+c_{6} z+2 c_{8} x\right) \\
& \left.\left(c_{4}+c_{6} x+c_{7} y+2 c_{10} z\right)^{2} \frac{d^{3} \Psi}{d u^{3}}\right)+i \mathrm{~h} c_{1}\left(c_{2}+\right. \\
& \left.\left.c_{5} y+c_{6} z+2 c_{8} x\right) \frac{d^{2} \Psi}{d u^{2}}\right]=0
\end{aligned}
$$

O grau do polinômio que define a variável auxiliar u depende da extensão da região de interesse em torno de um suposto sítio ativo de reação. Assim, devem ser testadas formulações correspondentes a polinômios de diferentes graus, a fim de verificar o potencial de aplicação de cada formulação resultante.

\section{Resultados e discussão}

A Figura 1 mostra o estado inicial de um sistema composto por átomos de Potássio $(\mathrm{K})$ e Flúor $(\mathrm{F})$, e as Figuras 2 a 6 esboçam a evolução temporal das isolinhas de potencial durante a formação da ligação iônica. Nesses mapas a rampa de cores varia linearmente entre $18 \mathrm{eV}$ (violeta) e zero eV (vermelho).
Observa-se que o formato das curvas de nível sofre alterações ao longo do tempo, indicando que as nuvens eletrônicas estão simultaneamente sofrendo deformação e sobreposição, características da formação de uma ligação.

Verifica-se também que o número de isolinhas envolvendo o átomo de Flúor aumenta ao longo do tempo, enquanto diminui o número de isolinhas envolvendo o átomo de Sódio, característica que revela o caráter iônico da ligação formada.

Do ponto de vista da química clássica, o átomo de sódio "cede um elétron" ao átomo de Flúor, formando um orbital molecular no qual o compartilhamento da nuvem vai se tornando progressivamente mais desigual.

\section{Legenda}
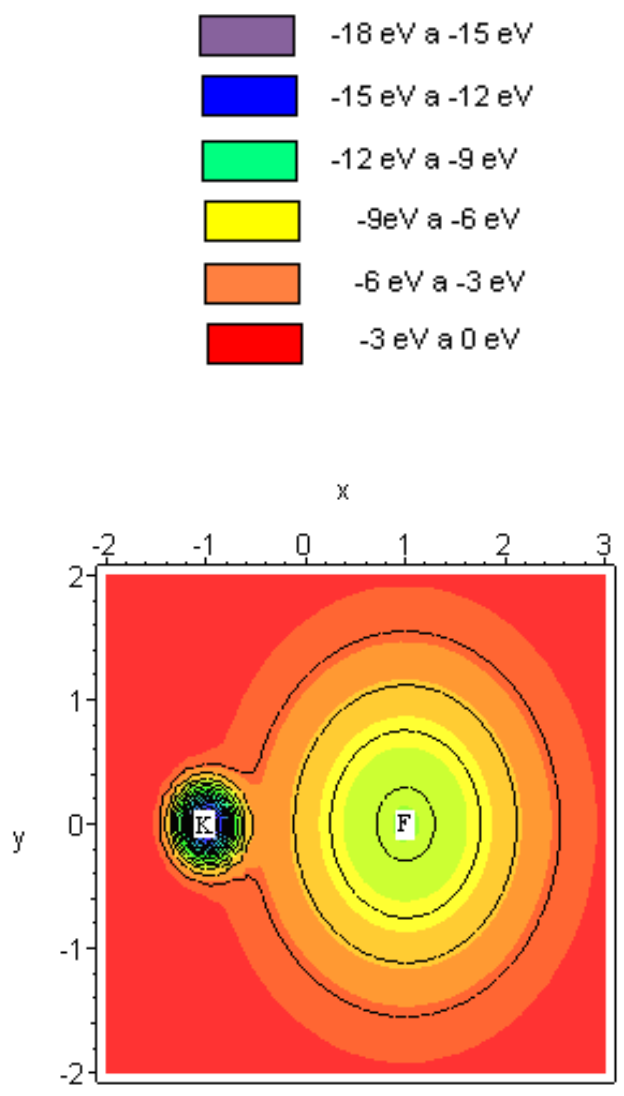

Figura 1. Isolinhas de potencial para o sistema $\mathrm{KF}(\mathrm{t}=0)$. 


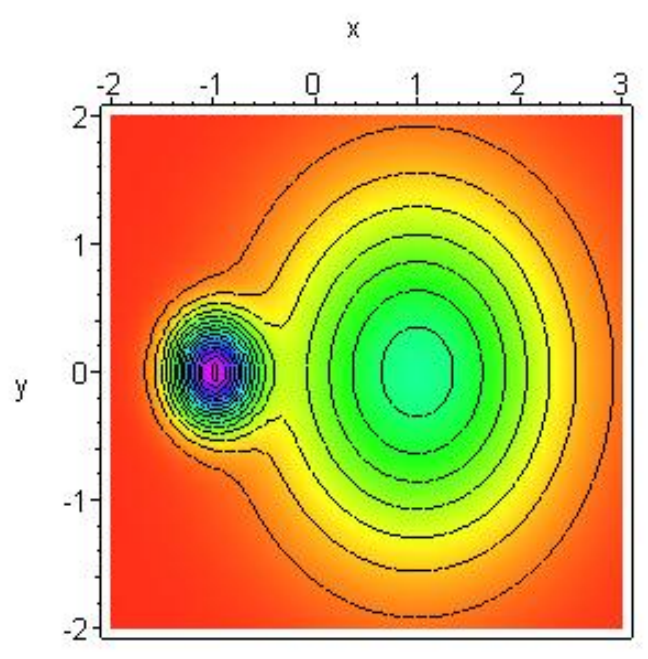

Figura 2. Isolinhas de potencial para o sistema $\mathrm{KF}(\mathrm{t}=20$ uat).

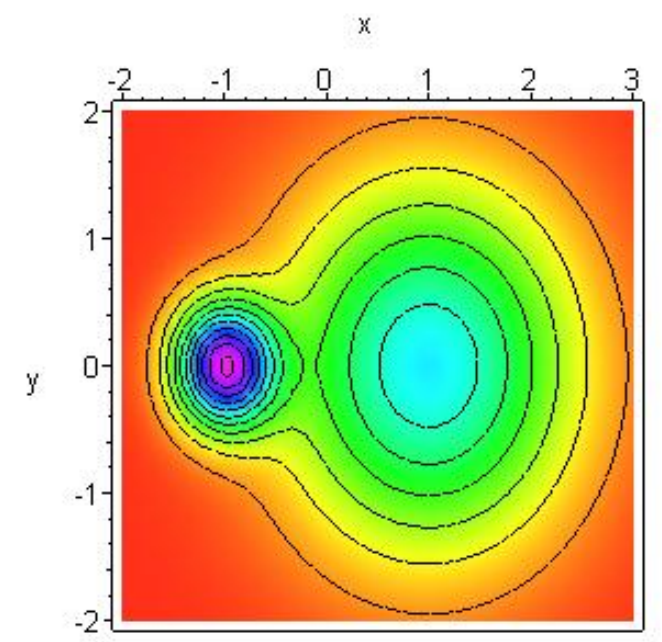

Figura 3. Isolinhas de potencial para o sistema KF ( $\mathrm{t}=40$ uat).

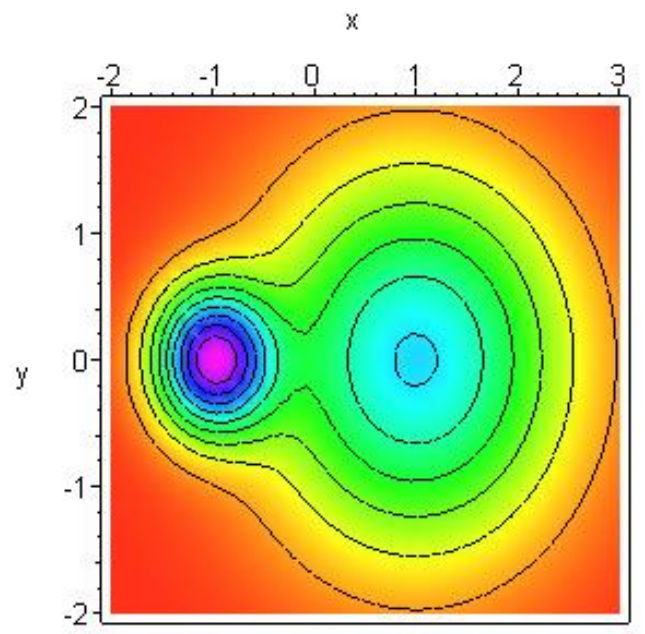

Figura 4. Isolinhas de potencial para o sistema KF $(t=60$ uat).

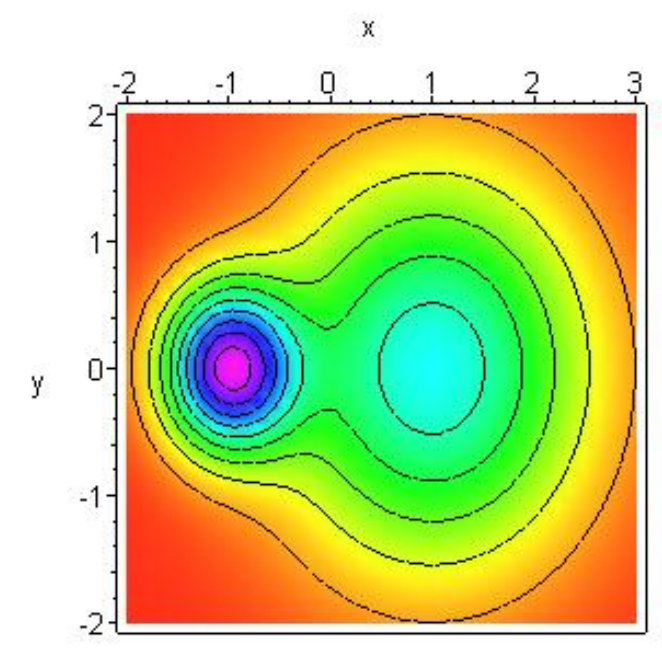

Figura 5. Isolinhas de potencial para o sistema KF (t=80 uat).

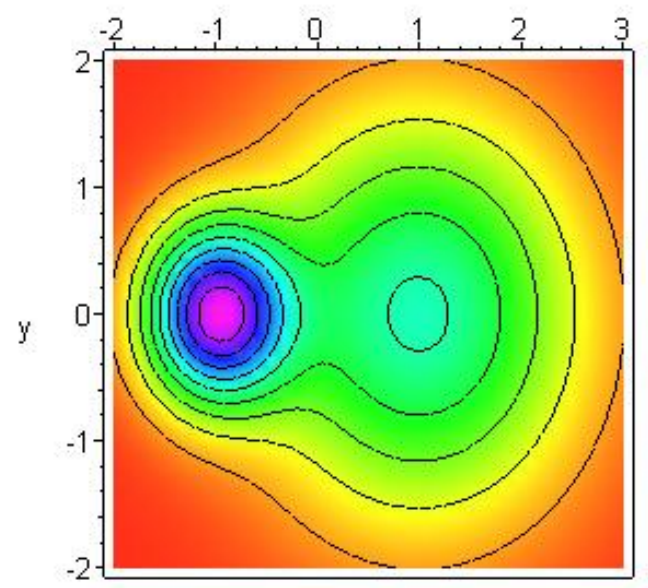

Figura 6. Isolinhas de potencial para o sistema KF ( $\mathrm{t}=100$ uat).

\section{Considerações Finais}

A reação entre o átomo de Flúor e o átomo de Potássio caracteriza a formação de uma ligação fortemente iônica. Quando uma ligação é covalente a densidade de isolinhas em torno de cada átomo envolvido é mais uniforme.

A simulação do processo de formação de ligações iônicas foi também efetuada utilizando outras combinações de metais alcalinos e alcalino-terrosos com halogênios e calcogênios, a fim de checar a consistência do modelo com resultados esperados, relativos à formação de óxidos e sais inorgânicos, de forma qualitativa. Em todos os exemplos testados, a proporção de caráter iônico aumentou com a diferença de eletronegatividade entre os elementos participantes da ligação, resultado consistente com o comportamento das moléculas inorgânicas.

Havendo constatado que a formulação foi bem sucedida no que diz respeito à consistência qualitativa 
com a dinâmica molecular esperada para a formação de ligações iônicas, os trabalhos futuros terão como foco a simulação de cenários mais complexos envolvendo reações entre moléculas orgânicas.

\section{Referências Bibliográficas}

[1] R. Daudel; R. Lefebvre; C. Moser, Quantum Chemistry Methods and Applications, Interscience Publisher, New York, 1959.

[2] A. Pullmann, Quantum Biochemistry, McGraw-Hill, New York, 1963.

[3] F. Pillar, Elementary Quantum Chemestry, McGraw-Hill, New York, 1968.

[4] H. Zimmerman, Quantum Mechanics for Organic Chemists, Academic Press, New York, 1975.

[5] J. P. Lowe, Quantum Chemistry, Academic Press, San Diego, 1978.

[6] J. Hutter, A. Curioni. "Car-Parrinello Molecular Dynamics on Massively Parallel Computers", ChemPhysChem, John Wiley \& Sons, Hoboken, NJ,2005, (6), pp. 1788-1793. 